\title{
A STUDY OF MEASURE-THEORETIC AREA FORMULAS
}

\author{
GIACOMO MARIA LECCESE AND VALENTINO MAGNANI
}

\begin{abstract}
AвsтRAct. We present a complete study of measure-theoretic area formulas in metric spaces, providing different measurability conditions.
\end{abstract}

\section{CONTENTS}

1. Introduction 1

2. Basic notions 3

3. Preliminary tools 5

4. Measure-theoretic area formulas 8

5. Applications to Hausdorff measures 12

References 19

\section{INTRODUCTION}

The problem of finding a proper notion of surface area has a long history in Calculus of Variations and in Geometric Measure Theory. Hausdorff measures, or more generally Carathéodory measures, are widely accepted as natural notions of surface area in metric spaces, [2, § 2.10.1]. On the other side, several important results of Geometric Measure Theory in Euclidean spaces are based on the fundamental concept of rectifiable set, whose Hausdorff measure can be computed by the classical area formula. Rectifiable sets can be considered in metric spaces, [2, \$3.2.14], and in relation to their density properties, a general metric area formula was established in [7], see also [1] for a different approach.

Substantial obstructions appear when we wish to compute the Hausdorff measure (or the spherical measure) of purely unrectifiable sets. An important family of such sets naturally appears in the geometries of homogeneous groups, [3], where also smooth submanifolds can be purely unrectifiable. This is a consequence of their Hausdorff dimension, that can be strictly greater than their topological dimension, [5, § 0.6.B]. In this framework, the use of measure-theoretic area formulas seems to be the only approach to find integral formulas for

Date: December 24, 2020.

2020 Mathematics Subject Classification. Primary 28A15. Secondary 28A78.

Key words and phrases. Differentiation of measures, spherical measure, Hausdorff measure, Carathéodory's contruction, area formula.

The author acknowledges the support of the University of Pisa, Project PRA 201849. 
the Hausdorff and spherical measure of submanifolds. In this connection, we mention some recent papers [4, 6, 9, 11, 12].

If the spherical measure is replaced by the centered Hausdorff measure, additional measuretheoretic area formulas have been proved in [4], leading to the relationship between perimeter measure and centered Hausdorff measure in stratified groups. In sum, applications to area formulas for submanifolds and to the perimeter measure in classes of homogeneous groups represent the first motivation of the present work. However, taking into account the deep relationship between density and rectifiability, [13, 14], there are also interesting connections with the so-called Besicovitch $\frac{1}{2}$-problem and rectifiability, discussed in [8, 10]. Considering that measure-theoretic area formulas are stated in general metric spaces, we would not be surprised about further applications.

We continue the study of measure-theoretic area formulas, according to which a suitable integration formula can be found between a Borel measure and a Carathéodory measure, [8]. The latter measure $\psi_{\zeta}$ is obtained by a gauge $\zeta: \mathcal{S} \rightarrow[0,+\infty]$ defined on a class of subsets $\mathcal{S} \subset \mathcal{P}(X)$ of a metric space $X$. When $\zeta$ is proportional to some real power $\alpha>0$ of the diameter of a set, the Carathéodory measure coincides with the Hausdorff measure $\mathcal{H}^{\alpha}$. In analogous way we obtain the spherical measure $\mathcal{S}^{\alpha}$ (Definition 2).

The general measure-theoretic area formula [8, Theorem 9] essentially arises from two key results: [2, Theorem 2.10.17(2)] and [2, Theorem 2.10.18(1)]. On the other hand, in considering a general $\zeta$, it may happen that the quotients $\mu(S) / \zeta(S)$ in the upper covering limits are not well defined for some $S \in \mathcal{S}$ of possibly small diameter. The values of $\zeta(S)$ may either vanish or be equal to $+\infty$ and the same values may be taken by $\mu(S)$. Another point in [2, Theorem 2.10.18(1)] is that the gauge $\zeta$ need not be necessarily defined on the enlargement $\hat{S}$ of $S$, see (2.7).

Revised versions of [2, Theorem 2.10.17(2)] and [2, Theorem 2.10.18(1)] are stated in [8] without proof. Actually, all the results of [8] are only stated. The previous two theorems can be extended to our Lemmas 3.1 and 3.2, hence leading in turn to two more general measuretheoretic area formulas, proved in Theorems 4.1 and 4.2. All of these results provide a reasonably complete picture of measure-theoretic area formulas for Carathéodory measures in metric spaces, giving indeed the second motivation for this work.

To define the Federer density, we introduce the quotient function $Q_{\mu, \zeta}$ with respect to a measure $\mu$ over $X$ and a gauge $\zeta$, (Definition 3). We restrict $Q_{\mu, \zeta}$ to a subclass $\mathcal{S}_{\mu, \zeta} \subset \mathcal{S}$ where it is unambiguously defined. It is somehow surprising the fact that, despite this restriction, the Federer density $F^{\zeta}(\mu, x)$, defined in (2.5) by $Q_{\mu, \zeta}$, still yields the following measure-theoretic area formula

$$
\mu(B)=\int_{B} F^{\zeta}(\mu, x) d \psi_{\zeta}(x)
$$

The cases where $\psi_{\zeta}$ is either the Hausdorff measure or the spherical measure is important for applications, where the Federer density has also more chances to be explicitly found. This fact has been shown for submanifolds or intrinsic submanifolds in different classes of noncommutative homogeneous groups, [4, 6, 9, 11, 12]. 
The Federer density $F^{\zeta}(\mu, \cdot)$ is denoted by $\mathfrak{D}^{\alpha}(\mu, \cdot)$ for the Hausdorff measure and by $\mathfrak{s}^{\alpha}(\mu, \cdot)$ for the spherical measure. In these cases it is also important to show that $\mathfrak{D}^{\alpha}(\mu, \cdot)$ and $\mathfrak{s}^{\alpha}(\mu, \cdot)$ are measurable (or Borel), but this is not straightforward. We mention that when the metric space is a homogeneous group and a suitable restriction of $\psi_{\zeta}$ is assumed to be locally doubling, the measurability of $\mathfrak{D}^{\alpha}(\mu, \cdot)$ and $\mathfrak{s}^{\alpha}(\mu, \cdot)$ is proved in [6, Proposition 2.3]. It is possible to show that in a metric space the Federer density $\mathfrak{D}^{\alpha}(\mu, \cdot)$ is Borel with respect to the subspace topology of a fixed set, according to Theorem 5.1. By a mild continuity assumption on the diameter function (Definition 4) and some more work, the previous Borel measurability can be extended to $\mathfrak{s}^{\alpha}(\mu, \cdot)$, see Theorem 5.6. Important consequences are the versions of (1.1) for the Hausdorff and spherical measure, established in Theorems 5.2, 5.3, 5.7 and 5.8.

\section{BASIC NOTIONS}

Throughout the paper, $X$ denotes a metric space equipped with a distance $d$. A covering relation is a subset $C$ of $\{(x, S): x \in S \in \mathcal{P}(X)\}$. Defining for $A \subset X$ the class

$$
C(A)=\{S: x \in A,(x, S) \in C\}
$$

we say that $C$ is fine at $x$, if for every $\epsilon>0$ there exists $S \in C(\{x\})$ such that diam $S<\epsilon$. Following the terminology of [2, §2.8.1], a nonempty family of sets $\mathcal{F} \subset \mathcal{P}(X)$ covers $A \subset X$ finely, if for each $a \in A$ and $\epsilon>0$ there exists $S \in \mathcal{F}$ with $a \in S \in \mathcal{F}$ such that $\operatorname{diam}(S)<\epsilon$. According to [2, $\$ 2.8 .16]$, the notion of covering relation yields the following notion of "upper" and "lower covering limits".

Definition 1 (Covering limits). If $C$ is a covering relation which is fine at $x \in X$,

$$
C(\{x\}) \subset \mathcal{D} \subset C(X)
$$

and $f: \mathcal{D} \rightarrow \overline{\mathbb{R}}$, then we define the upper and lower covering limit, respectively as

$$
\begin{aligned}
& \text { (C) } \limsup _{S \rightarrow x} f=\inf _{\epsilon>0} \sup \{f(S): S \in C(\{x\}), \operatorname{diam} S<\epsilon\}, \\
& (C) \liminf _{S \rightarrow x} f=\sup _{\epsilon>0} \inf \{f(S): S \in C(\{x\}), \operatorname{diam} S<\epsilon\} .
\end{aligned}
$$

The closed ball and the open ball of center $x \in X$ and radius $r>0$ are denoted by

$$
\mathbb{B}(x, r)=\{y \in X: d(x, y) \leq r\} \quad \text { and } B(x, r)=\{y \in X: d(x, y)<r\},
$$

respectively. The notion of $\delta$-covering and the Carathéodory construction of [2, §2.10.1] are both recalled in the next definition.

Definition 2. If $\delta>0, E \subset X$ and $\mathcal{E}=\left\{E_{j}: j \in \mathbb{N}\right\} \subset \mathcal{P}(X)$ is a covering of $E$ such that $\operatorname{diam}\left(E_{j}\right) \leq \delta$ for every $j \in \mathbb{N}$, we say that $\mathcal{E}$ is a $\delta$-covering of $E$. Let $\mathcal{S} \subset \mathcal{P}(X)$ and let $\zeta: \mathcal{S} \rightarrow[0,+\infty]$ be a fixed gauge. For $R \subset X$, we define

$$
\phi_{\zeta, \delta}(R)=\inf \left\{\sum_{j=0}^{\infty} \zeta\left(E_{j}\right):\left\{E_{j}: j \in \mathbb{N}\right\} \subset \mathcal{S} \text { is a } \delta \text {-covering of } R\right\} .
$$


The $\zeta$-approximating measure, or Carathéodory measure, is defined as follows

$$
\psi_{\zeta}(A)=\sup _{\delta>0} \phi_{\zeta, \delta}(A)
$$

for every $A \subset X$. Denoting by $\mathcal{F}$ the family of closed sets of $X$, for $\alpha, c_{\alpha}>0$, we define $\zeta_{\alpha}: \mathcal{F} \rightarrow[0,+\infty]$ by

$$
\zeta_{\alpha}(S)=c_{\alpha} \operatorname{diam}(S)^{\alpha} .
$$

Then the $\alpha$-dimensional Hausdorff measure is $\mathcal{H}^{\alpha}=\psi_{\zeta_{\alpha}}$. We define the set function

$$
\zeta_{b, \alpha}: \mathcal{F}_{b} \rightarrow[0,+\infty)
$$

as the restriction of $\zeta_{\alpha}$ to the family of all closed balls in $X$, that we denote by $\mathcal{F}_{b}$. Then $\psi_{\zeta_{b, \alpha}}$ is the $\alpha$-dimensional spherical Hausdorff measure, denoted by $\mathcal{S}^{\alpha}$.

We will use the terminology "measure over $X$ " to indicate an outer measure defined on all subsets of $X,[2, \S 2.1 .2]$. A gauge $\zeta: \mathcal{S} \rightarrow[0,+\infty]$ is also fixed on a nonempty class $\mathcal{S} \subset$ $\mathcal{P}(X)$. The next definition gives the notion of Federer density, introduced in [8, Definition 4].

Definition 3 (Federer density). Let $\mu$ be a measure over $X$ and let $\zeta: \mathcal{S} \rightarrow[0,+\infty]$ be a gauge. We introduce the subfamily of sets

$$
\mathcal{S}_{\mu, \zeta}=\mathcal{S} \backslash\{S \in \mathcal{S}: \zeta(S)=\mu(S)=0 \text { or } \mu(S)=\zeta(S)=+\infty\},
$$

along with the covering relation

$$
C_{\mu, \zeta}=\left\{(x, S): x \in S \in \mathcal{S}_{\mu, \zeta}\right\} .
$$

We choose $x \in X$ and assume that $C_{\mu, \zeta}$ is fine at $x$. We define the quotient function

$$
Q_{\mu, \zeta}: \mathcal{S}_{\mu, \zeta} \rightarrow[0,+\infty], \quad Q_{\mu, \zeta}(S)= \begin{cases}+\infty & \text { if } \zeta(S)=0 \\ \mu(S) / \zeta(S) & \text { if } 0<\zeta(S)<+\infty \\ 0 & \text { if } \zeta(S)=+\infty\end{cases}
$$

Then the Federer density, or upper $\zeta$-density of $\mu$ at $x \in X$, is well defined as

$$
F^{\zeta}(\mu, x)=\left(C_{\mu, \zeta}\right) \limsup _{S \rightarrow x} Q_{\mu, \zeta}(S) \text {. }
$$

We use a special notation when we consider Federer densities with respect to $\zeta_{\alpha}$ and $\zeta_{b, \alpha}$, respectively. If $\mu$ is a measure over $X$ and $C_{\mu, \zeta, \alpha}$ is fine at $x \in X$, then we set

$$
\mathfrak{s}^{\alpha}(\mu, x)=F^{\zeta b, \alpha}(\mu, x)
$$

If $C_{\mu, \zeta_{\alpha}}$ is fine at $x$, then we set

$$
\mathrm{D}^{\alpha}(\mu, x)=F^{\zeta_{\alpha}}(\mu, x) .
$$

These densities will appear in the measure-theoretic area formulas for the Hausdorff and spherical measure, see Section 5 . 
Remark 1. It is important to compute the upper limit (2.5) only over the sets $S \in C_{\mu, \zeta}$, even if the definition of $Q_{\mu, \zeta}$ in (2.4) could be extended to any subset. Let us consider the gauge $\zeta_{\alpha}: \mathcal{F} \rightarrow[0, \infty]$, with $\zeta_{\alpha}(A)=c_{\alpha} \operatorname{diam}(A)^{\alpha}$ for every $A \in \mathcal{F}$, where $\alpha>0$. If we extend the definition of $Q_{\mu, \zeta}$ to all of $\mathcal{F}$ using (2.4), then for every $x \in X$ it holds

$$
(\mathcal{F}) \limsup _{S \rightarrow x} Q_{\mu, \zeta}(S)=+\infty \text {. }
$$

Indeed, for every $\epsilon>0$, we have

$$
\sup \left\{Q_{\mu, \zeta}(S): S \in C(\{x\}), \operatorname{diam}(S)<\epsilon\right\}=Q_{\mu, \zeta}(\{x\})=+\infty .
$$

The upper covering limit (2.6) now differs from the Federer density $D(\mu, x)$. It cannot give the measure-theoretic area formulas of Theorems 5.2 and 5.3.

In the sequel, a number $\tau>1$ is fixed and we will use the notion of enlargement of $S$, namely we introduce the set

$$
\hat{S}=\bigcup\left\{T \in \mathcal{S}_{\mu, \zeta}: T \cap S \neq \emptyset \text { and } \operatorname{diam}(T) \leq \tau \operatorname{diam}(S)\right\}
$$

where $\mathcal{S}_{\mu, \zeta}$ is defined in (2.3).

\section{Preliminary tools}

As mentioned in the introduction, the notion of Federer's density based on the quotient function (2.4) differs from the upper covering limits appearing in [2, Theorem 2.10.17(2)] and [2, Theorem 2.10.18(1)]. The next lemmas show that in these theorems the Federer density (2.5) can replace the upper covering limits.

We will use the notion of regular measure taken from [2, §2.1.5].

Lemma 3.1. Let $\zeta: \mathcal{S} \rightarrow[0,+\infty]$ be a gauge and let $A \subset X$ be nonempty. We consider a measure $\mu$ over $X, t>0$ and assume that the following conditions hold.

(1) $\mu$ is a regular measure.

(2) $\mathcal{S}_{\mu, \zeta}$ covers A finely.

(3) For every $x \in A$ we have $F^{\zeta}(\mu, x)<t$.

Then $\mu(E) \leq t \psi_{\zeta}(E)$ for all $E \subset A$.

Proof. Let $B(\mu, t, \delta)$ be the set of all those points $x \in E$ such that whenever $S \in \mathcal{S}_{\mu, \zeta}$ and $x \in S$ with $\operatorname{diam}(S) \leq \delta$, we have

$$
\mu(E \cap S) \leq t \zeta(S)
$$

We claim that $E=\bigcup_{k=0}^{\infty} B\left(\mu, t, 2^{-k}\right)$. The hypothesis implies that for every fixed $x \in E$, there exists $\bar{k} \in \mathbb{N}$ such that for every $S \in \mathcal{S}_{\mu, \zeta}$ containing $x$ and $\operatorname{diam}(S) \leq 2^{-\bar{k}}$, we have

$$
Q_{\mu, \zeta}(S)<t
$$

There are two possible cases: $\zeta(S)=+\infty$ or

$$
0<\zeta(S)<+\infty \quad \text { and } \quad \frac{\mu(E \cap S)}{\zeta(S)} \leq \frac{\mu(S)}{\zeta(S)} \leq t .
$$


In any case $x \in B\left(\mu, t, 2^{-\bar{k}}\right)$ and the claim is proved. We notice that

$$
\mu\left(B\left(\mu, t, 2^{-k}\right)\right) \leq t \phi_{\zeta, 2^{-k}}\left(B\left(\mu, t, 2^{-k}\right)\right) .
$$

Indeed for every family $\left\{E_{i}\right\}_{i \in \mathbb{N}} \subset \mathcal{S}$ such that $\operatorname{diam}\left(E_{i}\right) \leq 2^{-k}$ and

$$
B\left(\mu, t, 2^{-k}\right) \subset \bigcup_{i \in \mathbb{N}} E_{i}
$$

we have

$$
\mu\left(B\left(\mu, t, 2^{-k}\right)\right) \leq \sum_{i \in \mathbb{N}} \mu\left(B\left(\mu, t, 2^{-k}\right) \cap E_{i}\right) \leq \sum_{i \in \mathbb{N}} \mu\left(E \cap E_{i}\right) \leq t \sum_{i \in \mathbb{N}} \zeta\left(E_{i}\right) .
$$

The last inequality holds in the case $E_{i} \in \mathcal{S}_{\mu, \zeta}$ for every $i$, where we use the definition of $B\left(\mu, t, 2^{-k}\right)$. It is also true for every $E_{i} \in \mathcal{S} \backslash \mathcal{S}_{\mu, \zeta}$, since in this case if $\mu\left(E_{i}\right)=\zeta\left(E_{i}\right)=+\infty$ the inequality is obvious, otherwise $\mu\left(E \cap E_{i}\right) \leq \mu\left(E_{i}\right)=\zeta\left(E_{i}\right)=0$. It follows that

$$
\begin{aligned}
\mu(E) & =\lim _{k \rightarrow \infty} \mu\left(B\left(\mu, t, 2^{-k}\right)\right) \leq t \limsup _{k \rightarrow \infty} \phi_{\zeta, 2^{-k}}\left(B\left(\mu, t, 2^{-k}\right)\right) \\
& \leq t \limsup _{k \rightarrow \infty} \psi_{\zeta}\left(B\left(\mu, t, 2^{-k}\right)\right) \leq t \psi_{\zeta}(E),
\end{aligned}
$$

where in the first equality we have used the assumption that $\mu$ is a regular measure.

An aspect to be clarified in [2, Theorem 2.10.18(1)] is that the gauge $\zeta$ in this theorem may not be necessarily defined on the enlargement $\hat{S}$. In the next lemma, the conditions (3.1) take into account this fact and the upper covering limit in [2, Theorem 2.10.18(1)] is replaced by the Federer density.

Lemma 3.2. Let $\mu$ be a measure over $X, B \subset X$ is nonempty and let $\zeta: \mathcal{S} \rightarrow[0,+\infty]$ be a gauge. We assume that the following conditions hold.

(1) $\mathcal{S}_{\mu, \zeta}$ is made by closed and $\mu$-measurable sets.

(2) $\mathcal{S}_{\mu, \zeta}$ covers B finely.

(3) We have $c \geq 1$ and $\eta>0$ such that for every $S \in \mathcal{S}_{\mu, \zeta}$ there exists $\tilde{S} \in \mathcal{S}$ such that

$$
\hat{S} \subset \tilde{S}, \quad \operatorname{diam}(\tilde{S}) \leq c \operatorname{diam}(S) \quad \text { and } \quad \zeta(\tilde{S}) \leq \eta \zeta(S),
$$

where $\hat{S}$ is the enlargement of $S$ defined in (2.7).

(4) Let $t>0$ be such that

$$
F^{\zeta}(\mu, x)>t
$$

for all $x \in B$.

Then for any open set $V$ containing $B$, we have $t \psi_{\zeta}(B) \leq \mu(V)$.

Proof. It is obviously not restrictive to assume $\mu(V)<+\infty$. For each $\delta>0$, we define

$$
\mathcal{T}_{\delta}=\left\{S \in \mathcal{S}_{\mu, \zeta}: t \zeta(S)<\mu(S), S \subset V \text { and } \operatorname{diam}(S) \leq \frac{\delta}{c}\right\} .
$$


We claim that $\mathcal{T}_{\delta}$ covers $B$ finely. We fix $x \in B$. Since $F^{\zeta}(\mu, x)>t$ and $V$ is open, there exists $S \in \mathcal{S}_{\mu, \zeta}$ such that $S \subset V$,

$$
\operatorname{diam}(S)<\frac{\delta}{c} \quad \text { and } \quad Q_{\mu, \zeta}(S)>t .
$$

Since $\zeta(S)<+\infty$, we have $\zeta(S)=0$ and $\mu(S)>0$, or $\zeta(S)>0$ and $Q_{\mu, \zeta}(S)=\mu(S) / \zeta(S)$. In both cases we have proved that $t \zeta(S)<\mu(S)$, so that the claim is established. In view of [2, Corollary 2.8.6], there exists a disjoint subfamily $\mathcal{G}$ of $\mathcal{T}_{\delta}$ such that

$$
B \backslash \bigcup \mathcal{H} \subset \bigcup\{\hat{S}: S \in \mathcal{G} \backslash \mathcal{H}\}
$$

for every finite class $\mathcal{H} \subset \mathcal{G}$. Since $\cup \mathcal{G} \subset V$ and $\mu(V)<+\infty$, the class $\mathcal{G}$ must be countable. Thus, given $\epsilon>0$, we can choose $\mathcal{H}_{\epsilon} \subset \mathcal{G}$ so that

$$
\sum_{S \in \mathcal{G} \backslash \mathcal{H}_{\epsilon}} \mu(S)<\epsilon
$$

We now use the hypothesis (3), choosing some $\tilde{S} \in \mathcal{S}$ such that conditions (3.1) hold for every $S \in \mathcal{G} \backslash \mathcal{H}_{\epsilon}$. Taking into account that $\mathcal{H}_{\epsilon} \cup\left\{\tilde{S} \subset X: S \in \mathcal{G} \backslash \mathcal{H}_{\epsilon}\right\}$ is still a $\delta$-cover of $B$, we have

$$
\phi_{\zeta, \delta}(B) \leq \sum_{S \in \mathcal{H}_{\epsilon}} \zeta(S)+\sum_{S \in \mathcal{G} \backslash \mathcal{H}_{\epsilon}} \zeta(\tilde{S})<t^{-1} \sum_{S \in \mathcal{H}_{\epsilon}} \mu(S)+\eta t^{-1} \epsilon \leq t^{-1}[\mu(V)+\eta \epsilon] .
$$

Since $\epsilon>0$ is independent of $\delta$, we let $\epsilon \rightarrow 0^{+}$, proving that $t \phi_{\zeta, \delta}(B) \leq \mu(V)$. The arbitrary choice of $\delta$ concludes the proof.

The previous lemmas imply the following characterization of the absolutely continuity between two measures.

Proposition 3.3. Let $\mu$ be a measure over $X$, let $A \subset X$ be nonempty and consider a gauge $\zeta: \mathcal{S} \rightarrow[0,+\infty]$. We assume that the following conditions hold.

(1) $\mu$ is a regular measure.

(2) Every element of $\mathcal{S}_{\mu, \zeta}$ is closed and $\mu$-measurable.

(3) $\mathcal{S}_{\mu, \zeta}$ covers A finely.

(4) A has a countable covering whose elements are open and have $\mu$-finite measure.

(5) We have $c \geq 1$ and $\eta>0$ such that for every $S \in \mathcal{S}_{\mu, \zeta}$ there exists $\tilde{S} \in \mathcal{S}$ such that

$$
\hat{S} \subset \tilde{S}, \quad \operatorname{diam}(\tilde{S}) \leq c \operatorname{diam}(S) \quad \text { and } \quad \zeta(\tilde{S}) \leq \eta \zeta(S),
$$

where $\hat{S}$ is the enlargement of $S$ defined in (2.7).

Then the condition $\mu \mathrm{L} A<<\psi_{\zeta}\llcorner A$ is equivalent to

$$
\mu\left(\left\{x \in A: F^{\zeta}(\mu, x)=+\infty\right\}\right)=0 .
$$

Proof. We first suppose that $\mu \mathrm{L} A<<\psi_{\zeta}\left\llcorner A\right.$. Let $\left\{V_{k}\right\}_{k \in \mathbb{N}}$ be a countable covering of $A$, whose elements are open and have $\mu$-finite measure. By Lemma 3.2, for every $t>0$ we have

$$
t \psi_{\zeta}\left(\left\{x \in A: F^{\zeta}(\mu, x)=+\infty\right\} \cap V_{k}\right) \leq \mu\left(V_{k}\right)<+\infty,
$$


so that $\psi_{\zeta}\left(\left\{x \in A: F^{\zeta}(\mu, x)=+\infty\right\} \cap V_{k}\right)=0$ for every $k \in \mathbb{N}$, hence

$$
\psi_{\zeta}\left(\left\{x \in A: F^{\zeta}(\mu, x)=+\infty\right\}\right)=0
$$

By our hypothesis, the equality (3.2) immediately follows.

Conversely, we assume that (3.2) holds and consider $B \subset A$ with $\psi_{\zeta}(B)=0$. We set

$$
B_{j}=\left\{x \in B: F^{\zeta}(\mu, x)<j\right\}
$$

and observe that $B=\bigcup_{j \geq 1} B_{j} \cup\left\{x \in B: F^{\zeta}(\mu, x)=+\infty\right\}$. By (3.2), it follows that

$$
\mu(B) \leq \sum_{j \geq 1} \mu\left(B_{j}\right) .
$$

Finally, we can apply Lemma 3.1 to obtain that

$$
\mu\left(B_{j}\right) \leq j \psi_{\zeta}\left(B_{j}\right) \leq j \psi_{\zeta}(B)=0
$$

for all $j \geq 1$, hence completing the proof.

Remark 2. It is important to observe that in Proposition 3.3 the absolute continuity between the two measures follows from (3.2) without assuming condition (4).

\section{Measure-theoretic area formulas}

This section is devoted to the proof of the measure-theoretic area formula under two different groups of assumptions. Combining Lemma 3.1, Lemma 3.2 and Proposition 3.3, we get a first version of the measure-theoretic area formula.

Theorem 4.1 (Measure-theoretic area formula I). Let $\mu$ be a measure over $X$ and consider a gauge $\zeta: \mathcal{S} \rightarrow[0,+\infty]$. We fix a nonempty set $A \subset X$ and assume that the following conditions hold.

(1) $\mu$ is both a regular measure and a Borel measure.

(2) Every element of $\mathcal{S}_{\mu, \zeta}$ is closed.

(3) $\mathcal{S}_{\mu, \zeta}$ covers A finely.

(4) A is a Borel set.

(5) A has a countable covering whose elements are open and have $\mu$-finite measure.

(6) We have $c \geq 1$ and $\eta>0$ such that for every $S \in \mathcal{S}_{\mu, \zeta}$ there exists $\tilde{S} \in \mathcal{S}$ such that

$$
\hat{S} \subset \tilde{S}, \quad \operatorname{diam}(\tilde{S}) \leq c \operatorname{diam}(S) \quad \text { and } \quad \zeta(\tilde{S}) \leq \eta \zeta(S),
$$

where $\hat{S}$ is the enlargement of $S$ defined in (2.7).

(7) The subset $\left\{x \in A: F^{\zeta}(\mu, x)=0\right\}$ is $\sigma$-finite with respect to $\psi_{\zeta}$.

(8) We have the absolute continuity $\mu \mathrm{L} A<<\psi_{\zeta} \mathrm{L} A$.

If $F^{\zeta}(\mu, \cdot): A \rightarrow[0,+\infty]$ is Borel, then for every Borel set $B \subset A$ we have

$$
\mu(B)=\int_{B} F^{\zeta}(\mu, x) d \psi_{\zeta}(x) .
$$


Proof. Let $A^{0}=\left\{x \in A: F^{\zeta}(\mu, x)=0\right\}$ be covered by a countable family $\left\{E_{k}\right\}_{k \in \mathbb{N}}$ of subsets such that $\psi_{\zeta}\left(E_{k}\right)<+\infty$ for every $k \in \mathbb{N}$. For $h>0$, applying Lemma 3.1, we get

$$
\mu\left(A^{0} \cap E_{k}\right) \leq h \psi_{\zeta}\left(A^{0} \cap E_{k}\right) \leq h \psi_{\zeta}\left(E_{k}\right)<+\infty .
$$

Letting $h \rightarrow 0^{+}$, we conclude that $\mu\left(A^{0}\right)=0$. We choose a Borel set $B \subset A$ and an arbitrary $\lambda \in(0,1)$, then we define

$$
B_{k}=\left\{x \in B: \lambda^{k+1}<F^{\zeta}(\mu, x) \leq \lambda^{k}\right\}
$$

for every $k \in \mathbb{Z}$, with $B_{+\infty}=\left\{x \in B: F^{\zeta}(\mu, x)=0\right\}$ and $B_{-\infty}=\left\{x \in B: F^{\zeta}(\mu, x)=+\infty\right\}$. These are all Borel sets. In particular, we have shown that replacing $B$ with $B_{+\infty}$ (4.1) holds. Proposition 3.3 shows that $\mu\left(\left\{x \in A: F^{\zeta}(\mu, x)=+\infty\right\}\right)=0$, therefore $\mu\left(B_{-\infty}\right)=0$. Since $\mu$ is Borel and assumption (5) holds, for an arbitrary $h>0$ we can find an open set $W_{h} \supset B_{-\infty}$ such that $\mu\left(W_{h} \backslash B_{-\infty}\right)=\mu\left(W_{h}\right)<h$. We apply Lemma3.2 to $B_{-\infty}$ with $t=1$, getting

$$
h>\mu\left(W_{h}\right)>\psi_{\zeta}\left(B_{-\infty}\right) .
$$

We conclude that $\psi_{\zeta}\left(B_{-\infty}\right)=0$ and (4.1) also holds with $B_{-\infty}$ in place of $B$. We are left to prove that (4.1) holds with $B$ replaced by $B^{\prime}=B \backslash\left(B_{+\infty} \cup B_{-\infty}\right)$. Using Lemma 3.1, for every $\epsilon>0$, we get

$$
\begin{aligned}
\mu\left(B^{\prime}\right)=\sum_{k \in \mathbb{Z}} \mu\left(B_{k}\right) & \leq \sum_{k \in \mathbb{Z}}(1+\epsilon) \lambda^{k} \psi_{\zeta}\left(B_{k}\right) \\
& =(1+\epsilon) \lambda^{-1} \sum_{k \in \mathbb{Z}} \lambda^{k+1} \psi_{\zeta}\left(B_{k}\right) \\
& \leq(1+\epsilon) \lambda^{-1} \int_{B^{\prime}} F^{\zeta}(\mu, x) d \psi_{\zeta}(x) .
\end{aligned}
$$

Taking into account the arbitrary choice of $\epsilon$ and $\lambda$, we have proved that

$$
\mu\left(B^{\prime}\right) \leq \int_{B^{\prime}} F^{\zeta}(\mu, x) d \psi_{\zeta}(x)
$$

To prove the opposite inequality, we consider the nontrivial case where $\mu\left(B^{\prime}\right)<+\infty$. Taking into account that $\mu$ is Borel and the assumption (5) holds, for every $\epsilon>0$ and every $k \in \mathbb{Z}$ there exists an open set $W_{k} \supseteq B_{k}$ such that $\mu\left(W_{k} \backslash B_{k}\right)<\epsilon 2^{-|k|}$. Since $\mu\left(B_{k}\right)<+\infty$ for all $k \in \mathbb{Z}$, it follows that

$$
\begin{aligned}
\mu\left(B^{\prime}\right) & =\sum_{k \in \mathbb{Z}} \mu\left(B_{k}\right)>\sum_{k \in \mathbb{Z}}\left(\mu\left(W_{k}\right)-\epsilon 2^{-|k|}\right) \\
& =\sum_{k \in \mathbb{Z}} \mu\left(W_{k}\right)-3 \epsilon .
\end{aligned}
$$

Using Lemma 3.2, we get

$$
\sum_{k \in \mathbb{Z}} \mu\left(W_{k}\right) \geq \sum_{k \in \mathbb{Z}} \lambda^{k+1} \psi_{\zeta}\left(B_{k}\right) \geq \lambda \int_{B^{\prime}} F^{\zeta}(\mu, x) d \psi_{\zeta}(x) .
$$


We have proved that

$$
\mu\left(B^{\prime}\right) \geq \lambda \int_{B^{\prime}} F^{\zeta}(\mu, x) d \psi_{\zeta}(x)-3 \epsilon
$$

The arbitrary choice of $\epsilon$ and $\lambda$ lead us to the opposite inequality

$$
\mu\left(B^{\prime}\right) \geq \int_{B^{\prime}} F^{\zeta}(\mu, x) d \psi_{\zeta}(x)
$$

hence concluding the proof.

Remark 3. We notice that the use of Proposition 3.3 in the proof of Theorem 4.1 depends on our choice to present the assumptions. In fact, if we replace the absolute continuity $\mu\left\llcorner A<<\psi_{\zeta}\llcorner A\right.$ with the condition

$$
\mu\left(\left\{x \in A: F^{\zeta}(\mu, x)=+\infty\right\}\right)=0,
$$

then Proposition 3.3 is not necessary in the proof.

In the next version of the measure-theoretic area formula, the Borel measurability of the Federer density is replaced by the more general $\psi_{\zeta}$-measurability. We can also consider a more general $\psi_{\zeta}$-measurable domain of integration $A$, requiring in addition the $\sigma$-finiteness with respect to $\psi_{\zeta}$.

Theorem 4.2 (Measure-theoretic area formula II). Let $\mu$ be a measure over $X$ and consider a gauge $\zeta: \mathcal{S} \rightarrow[0,+\infty]$. We fix a nonempty set $A \subset X$ and assume that the following conditions hold.

(1) $\mu$ is a Borel regular measure.

(2) Every element of $\mathcal{S}$ is Borel and every element of $\mathcal{S}_{\mu, \zeta}$ is closed.

(3) $\mathcal{S}_{\mu, \zeta}$ covers A finely.

(4) $A$ is $\psi_{\zeta}$-measurable and $\sigma$-finite with respect to $\psi_{\zeta}$.

(5) A has a countable covering whose elements are open and have $\mu$-finite measure.

(6) We have $c \geq 1$ and $\eta>0$ such that for every $S \in \mathcal{S}_{\mu, \zeta}$ there exists $\tilde{S} \in \mathcal{S}$ such that

$$
\hat{S} \subset \tilde{S}, \quad \operatorname{diam}(\tilde{S}) \leq c \operatorname{diam}(S) \quad \text { and } \quad \zeta(\tilde{S}) \leq \eta \zeta(S),
$$

where $\hat{S}$ is the enlargement of $S$ defined in (2.7).

(7) We have the absolute continuity $\mu \mathrm{L} A<<\psi_{\zeta} \mathrm{L} A$.

If $F^{\zeta}(\mu, \cdot): A \rightarrow[0,+\infty]$ is $\psi_{\zeta}$-measurable, then every $\psi_{\zeta}$-measurable subset $B \subset A$ is also $\mu$-measurable and the following formula holds

$$
\mu(B)=\int_{B} F^{\zeta}(\mu, x) d \psi_{\zeta}(x) .
$$

Proof. Let $B \subset A$ be a $\psi_{\zeta}$-measurable set. It is not restrictive to assume that $\psi_{\zeta}(B)<+\infty$. This is justified by the fact that $A$ can be written as a disjoint union $\bigcup_{i \in \mathbb{N}} A_{k}$, where $A_{k}$ is $\psi_{\zeta}$-measurable and it has $\psi_{\zeta}$-finite measure. Condition (2) implies that $\psi_{\zeta}$ is Borel regular, so even if we may have a priori a sequence of arbitrary sets that cover $A$ and have $\psi_{\zeta}$-finite 
measure, we can always assume they are Borel and in particular $\psi_{\zeta}$-measurable. Our claim for every $B \cap A_{k}$ implies that this set is $\mu$-measurable for every $k \in \mathbb{N}$ and

$$
\mu(B)=\sum_{k \in \mathbb{N}} \mu\left(B \cap A_{k}\right)=\sum_{k \in \mathbb{N}} \int_{B \cap A_{k}} F^{\zeta}(\mu, x) d \psi_{\zeta}(x)=\int_{B} F^{\zeta}(\mu, x) d \psi_{\zeta}(x) .
$$

The assumption that $\psi_{\zeta}(B)<+\infty$ in particular gives

$$
\psi_{\zeta}\left(\left\{x \in B: F^{\zeta}(\mu, x)=0\right\}\right)<+\infty .
$$

Since $\psi_{\zeta}$ is Borel regular and $\psi_{\zeta}(B)<+\infty$, we can write $B=B_{0} \cup N \subset A$, where $B_{0}$ is Borel and $\psi_{\zeta}(N)=0$. By our assumption, $\mu(N)=0$, hence $B$ is also $\mu$-measurable. Since $A$ is $\sigma$-finite with respect to $\psi_{\zeta}$, actually any $\psi_{\zeta}$-measurable subset of $A$ is also $\mu$-measurable.

We fix $0<\lambda<1$ and for every $k \in \mathbb{Z}$ define

$$
B_{k}=\left\{x \in B: \lambda^{k}<F^{\zeta}(\mu, x) \leq \lambda^{k+1}\right\},
$$

that is $\psi_{\zeta}$-measurable, along with

$$
B_{+\infty}=\left\{x \in B: F^{\zeta}(\mu, x)=0\right\} \quad \text { and } \quad B_{-\infty}=\left\{x \in B: F^{\zeta}(\mu, x)=+\infty\right\} .
$$

Thus, all of these sets are also $\mu$-measurable. Since $\psi_{\zeta}\left(B_{+\infty}\right)<+\infty$, for any fixed $\kappa>0$, due to Lemma 3.1, we get

$$
\mu\left(B_{+\infty}\right) \leq \kappa \psi_{\zeta}\left(B_{+\infty}\right)<+\infty .
$$

The arbitrary choice of $\kappa>0$ gives $\mu\left(B_{+\infty}\right)=0$. This proves (4.3) for $B=B_{+\infty}$. In view of Proposition 3.3 , we get

$$
\mu\left(\left\{x \in A: F^{\zeta}(\mu, x)=+\infty\right\}\right)=0
$$

and in particular $\mu\left(B_{-\infty}\right)=0$. We now use the fact that $\mu$ is Borel and assumption (5) holds. Then for any $h>0$ there exists an open set $W_{h} \supset B_{-\infty}$ such that $\mu\left(W_{h} \backslash B_{-\infty}\right)=\mu\left(W_{h}\right)<h$. By Lemma 3.2 with $t=1$, we get $h>\mu\left(W_{h}\right)>\psi_{\zeta}\left(B_{-\infty}\right)$, hence $\psi_{\zeta}\left(B_{-\infty}\right)=0$ and (4.1) holds with $B=B_{-\infty}$. We set $B^{\prime}=B \backslash\left(B_{+\infty} \cup B_{-\infty}\right)$ and observe that the validity of (4.3) for $B=B^{\prime}$ concludes the proof. Due to Lemma 3.1, for $\epsilon>0$ it holds

$$
\begin{aligned}
\mu\left(B^{\prime}\right)=\sum_{k \in \mathbb{Z}} \mu\left(B_{k}\right) & \leq \sum_{k \in \mathbb{Z}}(1+\epsilon) \lambda^{k} \psi_{\zeta}\left(B_{k}\right) \\
& \leq(1+\epsilon) \lambda^{-1} \sum_{k \in \mathbb{Z}} \lambda^{k+1} \psi_{\zeta}\left(B_{k}\right) \\
& \leq(1+\epsilon) \lambda^{-1} \int_{B^{\prime}} F^{\zeta}(\mu, x) d \psi_{\zeta}(x) .
\end{aligned}
$$

The arbitrary choice of $\epsilon$ and $\lambda$, give the first inequality

$$
\mu\left(B^{\prime}\right) \leq \int_{B^{\prime}} F^{\zeta}(\mu, x) d \psi_{\zeta}(x) .
$$

We now prove the opposite inequality, considering the nontrivial case where $\mu\left(B^{\prime}\right)<+\infty$. As before, the Borel regularity of $\mu$ and assumption (5) provide an outer approximation with 
open sets. For every $\epsilon>0$ and $k \in \mathbb{Z}$ there exists an open set $W_{k} \supseteq B_{k}$ such that

$$
\mu\left(W_{k} \backslash B_{k}\right)<\epsilon 2^{-|k|} .
$$

We obtain the following inequalities

$$
\mu\left(B^{\prime}\right)=\sum_{k \in \mathbb{Z}} \mu\left(B_{k}\right)>\sum_{k \in \mathbb{Z}}\left(\mu\left(W_{k}\right)-\epsilon 2^{-|k|}\right)=\sum_{k \in \mathbb{Z}} \mu\left(W_{k}\right)-3 \epsilon .
$$

Our assumptions also allow to apply Lemma 3.2, hence

$$
\sum_{k \in \mathbb{Z}} \mu\left(W_{k}\right) \geq \sum_{k \in \mathbb{Z}} \lambda^{k+1} \psi_{\zeta}\left(B_{k}\right) \geq \lambda \int_{B^{\prime}} F^{\zeta}(\mu, x) d \psi_{\zeta}(x) .
$$

It follows that

$$
\mu\left(B^{\prime}\right) \geq \lambda \int_{B^{\prime}} F^{\zeta}(\mu, x) d \psi_{\zeta}(x)-3 \epsilon .
$$

The arbitrary choice of $\epsilon>0$ and $\lambda$ provide the opposite inequality

$$
\mu\left(B^{\prime}\right) \geq \int_{B^{\prime}} F^{\zeta}(\mu, x) d \psi_{\zeta}(x)
$$

hence completing the proof.

Remark 4. We notice that in both Theorem 4.1 and Theorem 4.2, assuming that $\mu$ is finite on all open metric balls gives the assumption (5).

\section{Applications to Hausdorff measures}

The special cases where $\psi_{\zeta}$ is either the Hausdorff measure or the spherical measure are important for the applications in Geometric Measure Theory. The next theorems consider the case where the gauge is proportional to some power of the diameter. We prove that some of the "abstract conditions" for measure-theoretic area formulas are satisfied.

Theorem 5.1. Let $\mu$ be a measure over $X, \alpha>0$ and fix $A \subset X$. If $\mathcal{F}_{\mu, \zeta_{\alpha}}$ covers $A$ finely, then $\mathfrak{D}^{\alpha}(\mu, \cdot): A \rightarrow[0,+\infty]$ is Borel with respect to the subspace topology of $A$.

Proof. We have to prove that $\mathfrak{D}^{\alpha}(\mu, \cdot)$ is Borel with respect to the subspace topology of $A$. It suffices to prove that for any $\delta>0$ the function $\mathfrak{D}_{\delta}^{\alpha}(\mu, \cdot): A \rightarrow[0,+\infty]$ defined as

$$
D_{\delta}^{\alpha}(\mu, x)=\sup \left\{Q_{\mu, \zeta_{\alpha}}(S): x \in S, S \in \mathcal{F}_{\mu, \zeta_{\alpha}}, \operatorname{diam}(S)<\delta\right\}
$$

is Borel with respect to the subspace topology of $A$. We fix $t>0$ and consider the set

$$
\mathcal{S}_{t, \delta}=\left\{x \in A: \mathrm{D}_{\delta}^{\alpha}(\mu, x)>t\right\} .
$$

We wish to prove that $\mathcal{S}_{t, \delta}$ is open in $A$. Let us fix $y \in \mathcal{S}_{t, \delta}$. There exists a set $S \in \mathcal{F}_{\mu, \zeta_{\alpha}}$ containing $y$, such that

$$
Q_{\mu, \zeta_{\alpha}}(S)>t \quad \text { and } \quad \operatorname{diam}(S)<\delta
$$


If $\zeta_{\alpha}(S)=0$, then $S=\{y\}$ and $\mu(\{y\})>0$. We have only two possibilities: either there exists $\epsilon>0$ such that $\operatorname{diam}(\mathbb{B}(y, \epsilon))=0$ or we can find

$$
0<\epsilon<\min \left\{\frac{\delta}{2}, \frac{1}{2}\left(\frac{\mu(\{y\})}{t c_{\alpha}}\right)^{1 / \alpha}\right\}
$$

such that $\operatorname{diam}(\mathbb{B}(y, \epsilon))>0$. In the former case we get $Q_{\mu, \zeta_{\alpha}}(\mathbb{B}(y, \epsilon))=+\infty$ and we can also assume that $\epsilon<\delta / 2$. In the latter, we obtain

$$
Q_{\mu, \zeta_{\alpha}}(\mathbb{B}(y, \epsilon))=\frac{\mu(\mathbb{B}(y, \epsilon))}{c_{\alpha} \operatorname{diam}(\mathbb{B}(y, \epsilon))^{\alpha}} \geq \frac{\mu(\{y\})}{c_{\alpha}(2 \epsilon)^{\alpha}}>t .
$$

In both cases $\mathbb{B}(y, \epsilon) \in \mathcal{F}_{\mu, \zeta_{\alpha}}$, $\operatorname{diam}(\mathbb{B}(y, \epsilon))<\delta$ and

$$
Q_{\mu, \zeta_{\alpha}}(\mathbb{B}(y, \epsilon))>t .
$$

If we choose any $z \in \mathbb{B}(y, \epsilon)$, then the definition of Federer density gives

$$
t<Q_{\mu, \zeta^{\alpha}}(\mathbb{B}(y, \epsilon)) \leq \mathfrak{D}_{\delta}^{\alpha}(\mu, z) .
$$

We have shown that $B(y, \epsilon) \cap A \subset \mathcal{S}_{t, \delta}$. Now we consider the case $\zeta^{\alpha}(S)>0$, where

$$
Q_{\mu, \zeta^{\alpha}}(S)=\frac{\mu(S)}{\zeta^{\alpha}(S)}>t
$$

and $\operatorname{diam}(S)<\delta$. By the triangle inequality, since $y \in S$, there exists an $\epsilon>0$ such that

$$
\operatorname{diam}(S \cup \mathbb{B}(y, \epsilon)) \leq \operatorname{diam}(S)+2 \epsilon<\delta .
$$

Choosing a possibly smaller $\epsilon>0$, we also get

$$
\frac{\mu(S)}{c_{\alpha} \operatorname{diam}(S \cup \mathbb{B}(y, \epsilon))^{\alpha}}>t
$$

where $S \cup \mathbb{B}(y, \epsilon)$ is still closed and belongs to $\mathcal{F}_{\mu, \zeta_{\alpha}}$. We have proved that

$$
\frac{\mu(S \cup \mathbb{B}(y, \epsilon))}{\zeta^{\alpha}(S \cup \mathbb{B}(y, \epsilon))} \geq \frac{\mu(S)}{c_{\alpha} \operatorname{diam}(S \cup \mathbb{B}(y, \epsilon))^{\alpha}}>t .
$$

For every $z \in B(y, \epsilon) \cap A$, we obtain

$$
t<Q_{\mu, \zeta^{\alpha}}(S \cup \mathbb{B}(y, \epsilon)) \leq \mathfrak{D}_{\delta}^{\alpha}(\mu, z)
$$

hence $B(y, \epsilon) \cap A \subset \mathcal{S}_{t, \delta}$. Thus there exists an open set $\Omega$ such that $\mathcal{S}_{t, \delta}=\Omega \cap A$ and we may conclude that $\grave{D}_{\delta}^{\alpha}(\mu, \cdot)$ is lower semicontinuous on $A$ with respect to the subspace topology of $A$. Thus, $y \rightarrow \mathfrak{D}^{\alpha}(\mu, y)$ is Borel with respect to the subspace topology of $A$.

Remark 5. We denote by $\mathcal{F}^{\prime \prime}$ the family of closed bounded sets with positive diameter and observe that $\mathcal{F}^{\prime \prime}=\left(\mathcal{F}^{\prime \prime}\right)_{\mu, \zeta_{\alpha}}$. Then the assumption that $\mathcal{F}^{\prime \prime}$ covers $A$ finely implies that $(\mathcal{F})_{\mu, \zeta_{\alpha}}$ covers $A$ finely and from Theorem 5.1 we can conclude that $\mathfrak{D}^{\alpha}(\mu, \cdot)$ is well defined and Borel with respect to the subspace topology of $A$. If for every $x \in A$ there exists a sequence of closed bounded sets $F_{x, k}$ (depending on $x$ ) that contain $x$ and have positive diameter for every $k \in \mathbb{N}$, with $\operatorname{diam}\left(F_{x, k}\right) \rightarrow 0$, then $\mathcal{F}^{\prime \prime}$ covers $A$ finely and so does $(\mathcal{F})_{\mu, \zeta_{\alpha}}$ 
Theorem 5.2 (Area formula for the Hausdorff measure I). Let $\mu$ be a measure over $X, \alpha>0$ and fix $A \subset X$. We assume the validity of the following conditions.

(1) $\mu$ is both a regular measure and a Borel measure.

(2) $\mathcal{F}_{\mu, \zeta_{\alpha}}$ covers A finely.

(3) A is a Borel set.

(4) A has a countable covering whose elements are open and have $\mu$-finite measure.

(5) The subset $\left\{x \in A: \mathfrak{D}^{\alpha}(\mu, x)=0\right\}$ is $\sigma$-finite with respect to $\mathcal{H}^{\alpha}$.

(6) We have the absolute continuity $\mu \mathrm{L} A<<\mathcal{H}^{\alpha}\llcorner A$.

Then $\mathrm{D}^{\alpha}(\mu, \cdot): A \rightarrow[0,+\infty]$ is Borel and for every Borel set $B \subset A$ we have

$$
\mu(B)=\int_{B} \mathfrak{D}^{\alpha}(\mu, x) d \mathcal{H}^{\alpha}(x) .
$$

Proof. By Theorem 5.1 the function $\mathfrak{D}^{\alpha}(\mu, \cdot): A \rightarrow[0,+\infty]$ is Borel with respect to the subspace topology of $A$, hence it is also Borel. Condition (6) of Theorem 4.1 is satisfied by taking $\tilde{S}$ equal to the topological closure of $\hat{S}$, defined in (2.7). Thus, we are in the assumptions of Theorem 4.1 and our claim is established.

Theorem 5.3 (Area formula for the Hausdorff measure II). Let $\mu$ be a measure over $X, \alpha>0$ and fix $A \subset X$. We assume the validity of the following conditions.

(1) $\mu$ is a Borel regular measure.

(2) $\mathcal{F}_{\mu, \zeta_{\alpha}}$ covers A finely.

(3) A is $\mathcal{H}^{\alpha}$-measurable and $\sigma$-finite with respect to $\mathcal{H}^{\alpha}$.

(4) A has a countable covering whose elements are open and have $\mu$-finite measure.

(5) We have the absolute continuity $\mu \mathrm{L} A<<\mathcal{H}^{\alpha} \mathrm{L} A$.

Then $\mathrm{D}^{\alpha}(\mu, \cdot): A \rightarrow[0,+\infty]$ is $\mathcal{H}^{\alpha}$-measurable, every $\mathcal{H}^{\alpha}$-measurable set $B \subset A$ is also $\mu$-measurable and we have

$$
\mu(B)=\int_{B} \mathfrak{D}^{\alpha}(\mu, x) d \mathcal{H}^{\alpha}(x) .
$$

Proof. Theorem 5.1 shows that $\mathrm{D}^{\alpha}(\mu, \cdot): A \rightarrow[0,+\infty]$ is Borel with respect to the subspace topology of $A$. Since $A$ is $\mathcal{H}^{\alpha}$-measurable, we immediately observe that $\grave{D}^{\alpha}(\mu, \cdot)$ is also $\mathcal{H}^{\alpha}$ measurable. Condition (6) of Theorem 4.2 is satisfied by taking $\tilde{S}$ equal to the topological closure of $\hat{S}$, defined in (2.7). Thus, the application of Theorem 4.2 concludes the proof.

Remark 6. If $\mathcal{F}^{\prime \prime}$ is as in Remark [5, then in the assumptions of either Theorem 5.2 or Theorem 5.3 the collection $\mathcal{F}^{\prime \prime}$ covers $A$ finely if and only if so does $(\mathcal{F})_{\mu, \zeta_{\alpha}}$. As noticed in Remark 5, from the equality $\mathcal{F}^{\prime \prime}=\left(\mathcal{F}^{\prime \prime}\right)_{\mu, \zeta_{\alpha}} \subset(\mathcal{F})_{\mu, \zeta_{\alpha}}$, if $\mathcal{F}^{\prime \prime}$ covers $A$ finely clearly so does $(\mathcal{F})_{\mu, \zeta_{\alpha}}$. Conversely, if $(\mathcal{F})_{\mu, \zeta_{\alpha}}$ covers $A$ finely, then we fix any $x \in A$, getting a sequence of closed sets $\left\{F_{x, k}\right\} \subset(\mathcal{F})_{\mu, \zeta_{\alpha}}$ that all contain $x$ and such that $\operatorname{diam}\left(F_{x, k}\right) \rightarrow 0$. The absolute continuity of $\mu \mathrm{L} A$ with respect to $\mathcal{S}^{\alpha}\left\llcorner A\right.$ implies that $(\mathcal{F})_{\mu, \zeta_{\alpha}}$ contains no points. As a result, for all $k$ sufficiently large, we have $F_{x, k} \in \mathcal{F}^{\prime \prime}$. From the arbitrary choice of $x$ we infer that $\mathcal{F}^{\prime \prime}$ covers $A$ finely. 
The next area formulas will replace the Hausdorff measure with the spherical measure. This requires a minimal regularity condition on the diameter function.

Definition 4 (Diametric regularity). A metric space $X$ is called diametrically regular if for every $x \in X$ there exist an $R_{x}>0$ and a $\delta_{x}>0$ such that $\left(0, \delta_{x}\right) \ni r \rightarrow \operatorname{diam}(B(y, r))$ is a continuous function for every $y \in B\left(x, R_{x}\right)$.

Lemma 5.4. If a metric space $X$ is diametrically regular, then for every $x \in X$ there exist $R_{x}>0$ and $\delta_{x}>0$ such that whenever $0<t<\delta_{x}$ and $y \in B\left(x, R_{x}\right)$, we have

$$
\operatorname{diam}(B(y, t))=\operatorname{diam}(\mathbb{B}(y, t)) .
$$

Proof. Let $x \in X$ and choose $R_{x}$ and $\delta_{x}$ as in Definition 4 . For $0<t<\delta_{x}$, we consider any $t^{\prime} \in\left(t, \delta_{x}\right)$ and observe that $\operatorname{diam}(\mathbb{B}(y, t)) \leq \operatorname{diam}\left(B\left(y, t^{\prime}\right)\right)$ for all $y \in B\left(x, R_{x}\right)$. By diametric regularity, passing to the limit as $t^{\prime} \searrow t$, we obtain $\operatorname{diam}(\mathbb{B}(y, t)) \leq \operatorname{diam}(B(y, t))$, that concludes the proof.

Lemma 5.5. Let $\zeta_{o, \alpha}: \mathcal{F}_{o} \rightarrow[0,+\infty)$ be defined on the family $\mathcal{F}_{o}$ of all open balls of $X$ as $\zeta_{o, \alpha}(B)=c_{\alpha} \operatorname{diam}(B)^{\alpha}$ for every $B \in \mathcal{F}_{o}$. We fix $x \in X, \alpha>0$ and consider a measure $\mu$ over $X$. If $\left(\mathcal{F}_{b}\right)_{\mu, \zeta, \alpha}$ covers $\{x\}$ finely, then so does $\left(\mathcal{F}_{o}\right)_{\mu, \zeta_{o, \alpha}}$.

Proof. We consider an infinitesimal sequence of positive numbers $\epsilon_{k} \searrow 0$. By assumption, there exists a sequence of closed metric balls $\mathbb{B}\left(x_{k}, r_{k}\right) \in\left(\mathcal{F}_{b}\right)_{\mu, \zeta_{b, \alpha}}$ such that $x \in \mathbb{B}\left(x_{k}, r_{k}\right)$ and $\operatorname{diam}\left(\mathbb{B}\left(x_{k}, r_{k}\right)\right)<\epsilon_{k}$ for all $k \in \mathbb{N}$. We have only two possible cases. In the first one there exists $k_{0} \in \mathbb{N}$ such that $\operatorname{diam}\left(\mathbb{B}\left(x_{k}, r_{k}\right)\right)=0$ for all $k \geq k_{0}$. For these $k$ 's, we get $\mathbb{B}\left(x_{k}, r_{k}\right)=\{x\}$, hence $\mu(\{x\})>0$. If $\operatorname{diam}\left(B\left(x, \epsilon_{k} / 4\right)\right)>0$, then clearly $B\left(x, \epsilon_{k} / 4\right) \in\left(\mathcal{F}_{o}\right)_{\mu, \zeta_{o, \alpha}}$ and $\operatorname{diam}\left(B\left(x, \epsilon_{k} / 4\right)\right)<\epsilon_{k}$. In the remaining case, there exists a subsequence $r_{\alpha(k)}$ of $r_{k}$ such that $0<\operatorname{diam}\left(\mathbb{B}\left(x_{\alpha(k)}, r_{\alpha(k)}\right)\right)<\epsilon_{\alpha(k)}$ and $\mathbb{B}\left(x_{\alpha(k)}, r_{\alpha(k)}\right) \in\left(\mathcal{F}_{b}\right)_{\mu, \zeta_{b, \alpha}}$. We now fix any $k \in \mathbb{N}$. There exists $k_{1}>k$ such that

$$
0<2 \operatorname{diam}\left(\mathbb{B}\left(x_{\alpha\left(k_{1}\right)}, r_{\alpha\left(k_{1}\right)}\right)\right)<\operatorname{diam}\left(\mathbb{B}\left(x_{\alpha(k)}, r_{\alpha(k)}\right)\right)<\epsilon_{\alpha(k)}
$$

and $x \in \mathbb{B}\left(x_{\alpha\left(k_{1}\right)}, r_{\alpha\left(k_{1}\right)}\right)$. We observe that for every $y \in \mathbb{B}\left(x_{\alpha\left(k_{1}\right)}, r_{\alpha\left(k_{1}\right)}\right)$, we have

$$
d(y, x) \leq d\left(y, x_{\alpha\left(k_{1}\right)}\right)+d\left(x, x_{\alpha\left(k_{1}\right)}\right) \leq 2 \operatorname{diam}\left(\mathbb{B}\left(x_{\alpha\left(k_{1}\right)}, r_{\alpha\left(k_{1}\right)}\right)\right)<\epsilon_{\alpha(k)},
$$

therefore $\mathbb{B}\left(x_{\alpha\left(k_{1}\right)}, r_{\alpha\left(k_{1}\right)}\right) \subset B\left(x, \epsilon_{\alpha(k)}\right)$. We have proved that $\operatorname{diam}\left(B\left(x, \epsilon_{\alpha(k)}\right)\right)>0$ for every $k \in \mathbb{N}$, hence $B\left(x, \epsilon_{\alpha(k)}\right) \in\left(\mathcal{F}_{o}\right)_{\mu, \zeta_{o, \alpha}}$. In any of the two cases $\left(\mathcal{F}_{o}\right)_{\mu, \zeta_{o, \alpha}}$ covers $\{x\}$ finely.

Theorem 5.6. Let $X$ be diametrically regular, $\alpha>0$ and let $\mu$ be a measure over $X$. If $A \subset X$ and $\left(\mathcal{F}_{b}\right)_{\mu, \zeta b, \alpha}$ covers $A$ finely, then $\mathfrak{s}^{\alpha}(\mu, \cdot): A \rightarrow[0,+\infty]$ is Borel with respect to the subspace topology of $A$.

Proof. We consider the gauge $\zeta_{o, \alpha}$ of Lemma 5.5. The lemma shows that in our assumptions also $\left(\mathcal{F}_{o}\right)_{\mu, \zeta_{o, \alpha}}$ covers $A$ finely, so also the Federer density $F^{\zeta_{o, \alpha}}(\mu, x)$ is well defined at each point $x \in A$. Our first claim is the following equality

$$
\mathfrak{s}^{\alpha}(\mu, x)=F^{\zeta_{o, \alpha}}(\mu, x)
$$


for every $x \in A$. We fix $x \in A$ and consider $R_{x}>0$ and $\delta_{x}>0$ of Definition 4. We wish to prove that

$$
F^{\zeta o, \alpha}(\mu, x) \leq \mathfrak{s}^{\alpha}(\mu, x)
$$

It is equivalent to showing that

$$
\inf _{\delta>0} \sup _{\substack{x \in B \in\left(\mathcal{F}_{o}\right)_{\mu, \zeta o, \alpha} \\ \operatorname{diam}(B)<\delta}} Q_{\mu, \zeta_{o, \alpha}}(B) \leq \inf _{\delta>0} \sup _{\substack{x \in B \in\left(\mathcal{F}_{b}\right)_{\mu, \zeta b, \alpha} \\ \operatorname{diam}(B)<\delta}} Q_{\mu, \zeta_{b, \alpha}}(B) .
$$

We fix $\delta>0$ such that $\delta<\min \left\{R_{x}, \delta_{x}\right\}$ and choose $B \in\left(\mathcal{F}_{o}\right)_{\mu, \zeta_{o, \alpha}}$ such that $x \in B$ and $\operatorname{diam}(B)<\delta$. We may write $B=B(y, r)$ for some $y \in X$ and $r>0$. We define

$$
t_{y}=\sup \{d(y, z): z \in B\} \leq r
$$

and notice that clearly $B \subset \mathbb{B}\left(y, t_{y}\right)$, hence

$$
\mu(B) \leq \mu\left(\mathbb{B}\left(y, t_{y}\right)\right) .
$$

We observe that $t_{y} \leq r$ and

$$
t_{y} \leq \operatorname{diam}(B)<\delta<\min \left\{R_{x}, \delta_{x}\right\} .
$$

In particular, $y \in B\left(x, R_{x}\right)$ and $t_{y}<\delta_{x}$. Since $B \subset \mathbb{B}\left(y, t_{y}\right) \subset B\left(y, t_{y}^{\prime}\right)$ for every $t_{y}^{\prime}>t_{y}$, then $\operatorname{diam}(B) \leq \operatorname{diam}\left(B\left(y, t_{y}^{\prime}\right)\right)$ and by diametric regularity for $t_{y}^{\prime} \searrow t_{y}$ and $t_{y}^{\prime}<\delta_{x}$, we obtain

$$
\operatorname{diam}(B) \leq \operatorname{diam}\left(B\left(y, t_{y}\right)\right) \text {. }
$$

Moreover $B\left(y, t_{y}\right) \subset B(y, r)$ implies that

$$
\operatorname{diam}\left(B\left(y, t_{y}\right)\right) \leq \operatorname{diam}(B(y, r)) .
$$

By inequalities (5.6), (5.7) and by Lemma 5.4 applied to the ball $B\left(y, t_{y}\right)$, we obtain

$$
\operatorname{diam}(B)=\operatorname{diam}\left(\mathbb{B}\left(y, t_{y}\right)\right) .
$$

If $\operatorname{diam}(B)>0$, by equation (5.8) we have $\mathbb{B}\left(y, t_{y}\right) \in\left(\mathcal{F}_{b}\right)_{\mu, \zeta b, \alpha}$ and using (5.5) and again equation (5.8), we obtain

$$
Q_{\mu, \zeta_{o, \alpha}}(B)=\frac{\mu(B)}{c_{\alpha} \operatorname{diam}(B)^{\alpha}} \leq \frac{\mu\left(\mathbb{B}\left(y, t_{y}\right)\right)}{c_{\alpha} \operatorname{diam}\left(\mathbb{B}\left(y, t_{y}\right)\right)^{\alpha}}=Q_{\mu, \zeta b, \alpha}\left(\mathbb{B}\left(y, t_{y}\right)\right) .
$$

If $\operatorname{diam}(B(y, r))=0$, taking into account that $B \in\left(\mathcal{F}_{o}\right)_{\mu, \zeta_{o, \alpha}}$, we have $\mu(B(y, r))>0$. In addition, $B(y, r)=\mathbb{B}\left(y, \frac{r}{2}\right)=\{y\}=\{x\}$ and $\operatorname{diam}\left(\mathbb{B}\left(x, \frac{r}{2}\right)\right)=0$, hence

$$
Q_{\mu, \zeta_{o, \alpha}}(B(y, r))=Q_{\mu, \zeta_{b, \alpha}}\left(\mathbb{B}\left(y, \frac{r}{2}\right)\right)=+\infty .
$$

We have proved that for every $\delta>0$ such that $\delta<\min \left\{R_{x}, \delta_{x}\right\}$ the inequality (5.4) holds.

To prove the opposite inequality, we first consider the case $\mathfrak{s}^{\alpha}(\mu, x)=+\infty$. Then we can find a family of closed balls $\left\{C_{k}\right\}_{k \in \mathbb{N}} \subset\left(\mathcal{F}_{b}\right)_{\mu, \zeta_{b, \alpha}}$ such that, for every $k \in \mathbb{N}$ we have $\operatorname{diam}\left(C_{k}\right)<2^{-k}, x \in C_{k}$ and

$$
\lim _{k \rightarrow+\infty} Q_{\mu, \zeta b, \alpha}\left(C_{k}\right)=+\infty
$$


If there exists $k_{0} \in \mathbb{N}$ such that for every $k \in \mathbb{N}$ with $k \geq k_{0}$ we have $\operatorname{diam}\left(C_{k}\right)>0$, then there exists $\tilde{r}_{k}>0$ such that $C_{k}=\mathbb{B}\left(x_{k}, \tilde{r}_{k}\right)$ and the following supremum satisfies

$$
r_{k}=\sup \left\{d\left(z, x_{k}\right): z \in C_{k}\right\}>0 \text { and } r_{k} \leq \tilde{r}_{k} .
$$

We have seen that $\mathbb{B}\left(x_{k}, r_{k}\right) \subset C_{k}$. On the other hand, by definition of $r_{k}$ we also get

$$
C_{k} \subset \mathbb{B}\left(x_{k}, r_{k}\right),
$$

hence $C_{k}=\mathbb{B}\left(x_{k}, r_{k}\right)$. We select any $r_{k}^{\prime} \in\left(r_{k}, 2 r_{k}\right]$ and observe that

$$
0<\operatorname{diam}\left(C_{k}\right) \leq \operatorname{diam}\left(B\left(x_{k}, r_{k}^{\prime}\right)\right) \leq 2 r_{k}^{\prime} \leq 4 r_{k} \leq 4 \operatorname{diam}\left(C_{k}\right)
$$

where the last inequality follows from the definition of $r_{k}$. It follows that

$$
\frac{\mu\left(B\left(x_{k}, r_{k}^{\prime}\right)\right)}{\zeta_{o, \alpha}\left(B\left(x_{k}, r_{k}^{\prime}\right)\right)} \geq 4^{-\alpha} \frac{\mu\left(C_{k}\right)}{\zeta_{b, \alpha}\left(C_{k}\right)}=4^{-\alpha} Q_{\mu, \zeta b, \alpha}\left(C_{k}\right) \rightarrow+\infty
$$

as $k \rightarrow+\infty$. This esablishes the opposite inequality when the diameters of $C_{k}$ are positive for all large $k$ 's. In the remaining case there exists a strictly increasing sequence of integers $\alpha(k) \in \mathbb{N}$ such that such that $\operatorname{diam}\left(C_{\alpha(k)}\right)=0$ for all $k \in \mathbb{N}$. It follows that $C_{\alpha(k)}=\{x\}$ and $\mu(\{x\})>0$. We choose any deacreasing sequence $s_{k} \searrow 0$, hence

$$
\limsup _{k \rightarrow+\infty} \frac{\mu\left(B\left(x, s_{k}\right)\right)}{\zeta^{o, \alpha}\left(B\left(x, s_{k}\right)\right)} \geq \limsup _{k \rightarrow+\infty} \frac{\mu(\{x\})}{c_{\alpha} \operatorname{diam}\left(B\left(x, s_{k}\right)\right)^{\alpha}} \geq \limsup _{k \rightarrow+\infty} \frac{\mu(\{x\})}{c_{\alpha}\left(2 s_{k}\right)^{\alpha}}=+\infty .
$$

We have shown that $F^{\zeta_{o, \alpha}}(\mu, x)=+\infty$, hence concluding the proof of the inequality

$$
\mathfrak{s}^{\alpha}(\mu, x) \leq F^{\zeta o, \alpha}(\mu, x)
$$

in the case $\mathfrak{s}^{\alpha}(\mu, x)=+\infty$. We will prove (5.9) when $\mathfrak{s}^{\alpha}(\mu, x)<+\infty$. It is convenient to restrict our attention to the following subset of integers

$$
\Lambda=\left\{k \in \mathbb{N}: 2^{-k}<\min \left\{R_{x}, \delta_{x}\right\}\right\},
$$

where $R_{x}$ and $\delta_{x}$ are those of Definition 4 , By our assumptions, there exists a family

$$
\left\{D_{k}: k \in \Lambda\right\} \subset\left(\mathcal{F}_{b}\right)_{\mu, \zeta b, \alpha}
$$

of closed balls such that $x \in D_{k}$ for all $k \in \mathbb{N}$ and there exists $k_{1} \in \mathbb{N}$ such that for every $k \in \Lambda$ with $k \geq k_{1}$ we have $\operatorname{diam}\left(D_{k}\right)<2^{-k}$ and

$$
\sup \left\{Q_{\mu, \zeta^{b, \alpha}}(D): x \in D, D \in\left(\mathcal{F}_{b}\right)_{\mu, \zeta_{b, \alpha}}, \operatorname{diam}(D)<2^{-k}\right\}-2^{-k}<Q_{\mu, \zeta_{b, \alpha}}\left(D_{k}\right)<+\infty .
$$

As a consequence, for these $k$ 's we have that $\zeta_{b, \alpha}\left(D_{k}\right)>0$ and

$$
Q_{\mu, \zeta b, \alpha}\left(D_{k}\right)=\frac{\mu\left(D_{k}\right)}{\zeta_{b, \alpha}\left(D_{k}\right)} .
$$

We choose any $k \in \Lambda$ with $k \geq k_{0}$. Arguing as before, we find a center $y_{k} \in D_{k}$ and the following positive radius

$$
t_{k}=\sup \left\{d\left(z, y_{k}\right): z \in D_{k}\right\} \leq \operatorname{diam}\left(D_{k}\right)<2^{-k}<\delta_{x}
$$


such that $D_{k}=\mathbb{B}\left(y_{k}, t_{k}\right)$. We know that $d\left(y_{k}, x\right) \leq \operatorname{diam}\left(D_{k}\right)<2^{-k}<R_{x}$, therefore the diametric regularity and Lemma 5.4 imply that $s \rightarrow \operatorname{diam}\left(B\left(y_{k}, s\right)\right)=\operatorname{diam}\left(\mathbb{B}\left(y_{k}, s\right)\right)$ is continuous on $\left(0, \delta_{x}\right)$. Then we can find $t_{k}^{\prime} \in\left(t_{k}, \delta_{x}\right)$ such that $0<\operatorname{diam}\left(B\left(y_{k}, t_{k}^{\prime}\right)\right)<2^{-k}$ and

$$
\frac{\mu\left(D_{k}\right)}{\zeta_{b, \alpha}\left(D_{k}\right)}-2^{-k}<\frac{\mu\left(D_{k}\right)}{\zeta_{o, \alpha}\left(B\left(y_{k}, t_{k}^{\prime}\right)\right)} \leq \frac{\mu\left(B\left(y_{k}, t_{k}^{\prime}\right)\right)}{\zeta_{o, \alpha}\left(B\left(y_{k}, t_{k}^{\prime}\right)\right)} \text {. }
$$

We have proved that

$$
Q_{\mu, \zeta_{b, \alpha}}\left(D_{k}\right)-2^{-k} \leq \sup \left\{Q_{\mu, \zeta^{b, \alpha}}(B): x \in B, B \in\left(\mathcal{F}_{o}\right)_{\mu, \zeta_{o, \alpha}}, \operatorname{diam}(B)<2^{-k}\right\},
$$

for all $k \in \Lambda$ with $k \geq k_{1}$. As $k \rightarrow+\infty$, the opposite (5.9) follows and the claim (5.3) is established. Thus, the proof is finished if we show that $F^{\zeta_{o, \alpha}}: A \rightarrow[0,+\infty]$ is Borel with respect to the subspace topology of $A$. It suffices to show such measurability for $F_{\delta}^{\zeta_{o, \alpha}}(\mu, \cdot)$ : $A \rightarrow[0,+\infty]$ defined as follows

$$
F_{\delta}^{\zeta_{o, \alpha}}(\mu, x)=\sup \left\{Q_{\mu, \zeta_{o, \alpha}}(B): x \in B, B \in\left(\mathcal{F}_{o}\right)_{\mu, \zeta_{o, \alpha}}, \operatorname{diam}(\mathrm{S})<\delta\right\}
$$

when $\delta>0$ is arbitrarily chosen. We fix $t>0$ and consider the set

$$
\mathcal{S}_{t, \delta}=\left\{z \in A: F_{\delta}^{\zeta o, \alpha}(\mu, z)>t\right\} .
$$

If $y \in \mathcal{S}_{t, \delta}$, then we can find an open ball $B \in\left(\mathcal{F}_{o}\right)_{\mu, \zeta_{o, \alpha}}$ such that $x \in B$, $\operatorname{diam}(B)<\delta$ and $Q_{\mu, \zeta_{o, \alpha}}(B)>t$. For every $z \in B \cap A$, we have

$$
F_{\delta}^{\zeta o, \alpha}(\mu, z) \geq Q_{\mu, \zeta^{o, \alpha}}(B)>t
$$

so that $z \in \mathcal{S}_{t, \delta}$, hence $B \cap A \subset \mathcal{S}_{t, \delta}$. This shows that $\mathcal{S}_{t, \delta}$ is open in the subspace topology of $A$ and in particular it is Borel with respect to this topology.

Remark 7. If we denote by $\mathcal{F}_{b}^{\prime}$ the family of closed balls with positive diameter, then clearly $\mathcal{F}_{b}^{\prime}=\left(\mathcal{F}_{b}^{\prime}\right)_{\mu, \zeta_{b, \alpha}}$. As a result, if $\mathcal{F}_{b}^{\prime}$ covers $A$ finely, then $\left(\mathcal{F}_{b}\right)_{\mu, \zeta_{b, \alpha}}$ also covers $A$ finely and the Federer density $\mathfrak{s}^{\alpha}(\mu, \cdot)$ of Theorem 5.6 is well defined. For instance, if for every $x \in A$ there exists a sequence of closed metric balls $\mathbb{B}_{x, k}$ (depending on $x$ ) with positive diameter and containing $x$, such that $\operatorname{diam}\left(\mathbb{B}_{x, k}\right) \rightarrow 0$, then $\mathcal{F}_{b}^{\prime}$ covers $A$ finely and so does $(\mathcal{F})_{\mu, \zeta_{b, \alpha}}$.

Theorem 5.7 (Area formula for the spherical measure I). Let $\mu$ be a measure over $X, \alpha>0$ and fix $A \subset X$. We assume the validity of the following conditions.

(1) $X$ is a diametrically regular metric space.

(2) $\mu$ is both a regular measure and a Borel measure.

(3) $\left(\mathcal{F}_{b}\right)_{\mu, \zeta_{b, \alpha}}$ covers A finely.

(4) A is a Borel set.

(5) A has a countable covering whose elements are open and have $\mu$-finite measure.

(6) The subset $\left\{x \in A: \mathfrak{s}^{\alpha}(\mu, x)=0\right\}$ is $\sigma$-finite with respect to $\mathcal{S}^{\alpha}$.

(7) We have the absolute continuity $\mu\left\llcorner A<<\mathcal{S}^{\alpha}\llcorner A\right.$.

Then $\mathfrak{s}^{\alpha}(\mu, \cdot): A \rightarrow[0,+\infty]$ is Borel and for every Borel set $B \subset A$ we have

$$
\mu(B)=\int_{B} \mathfrak{s}^{\alpha}(\mu, x) d \mathcal{S}^{\alpha}(x)
$$


Proof. Due to Theorem 5.6 the density $\mathfrak{s}^{\alpha}(\mu, \cdot): A \rightarrow[0,+\infty]$ is Borel with respect to the subspace topology of $A$, hence by our assumptions it is also Borel. If $D_{0}=\mathbb{B}(x, r)$ is any closed ball, we consider its enlargement

$$
\widehat{D_{0}}=\bigcup\left\{D \in\left(\mathcal{F}_{b}\right)_{\mu, \zeta b, \alpha}: D \cap D_{0} \neq \emptyset \text { and } \operatorname{diam}(D) \leq \tau \operatorname{diam}\left(D_{0}\right)\right\},
$$

according to 2.7). Then the closed ball $\widetilde{D_{0}}=\mathbb{B}\left(x,(1+\tau) \operatorname{diam}\left(D_{0}\right)\right)$ contains $\widehat{D}_{0}$ and satisfies condition (6) of Theorem 4.1. All assumptions of Theorem 4.1 are satisfied and this leads us to the conclusion.

Theorem 5.8 (Area formula for the spherical measure II). Let $\mu$ be a measure over $X, \alpha>0$ and fix $A \subset X$. We assume the validity of the following conditions.

(1) $X$ is a diametrically regular metric space.

(2) $\mu$ is a Borel regular measure.

(3) $\left(\mathcal{F}_{b}\right)_{\mu, \zeta_{b, \alpha}}$ covers A finely.

(4) $A$ is $\mathcal{S}^{\alpha}$-measurable and $\sigma$-finite with respect to $\mathcal{S}^{\alpha}$.

(5) A has a countable covering whose elements are open and have $\mu$-finite measure.

(6) We have the absolute continuity $\mu \mathrm{L} A<<\mathcal{S}^{\alpha}\llcorner A$.

Then $\mathfrak{s}^{\alpha}(\mu, \cdot): A \rightarrow[0,+\infty]$ is $\mathcal{S}^{\alpha}$-measurable, every $\mathcal{S}^{\alpha}$-measurable set $B \subset A$ is also $\mu$-measurable and we have

$$
\mu(B)=\int_{B} \mathfrak{s}^{\alpha}(\mu, x) d \mathcal{S}^{\alpha}(x) .
$$

Proof. In view of Theorem 5.6, the density $\mathfrak{s}^{\alpha}(\mu, \cdot): A \rightarrow[0,+\infty]$ is Borel with respect to the subspace topology of $A$. Being $A$ an $\mathcal{S}^{\alpha}$-measurable set, then $\mathfrak{s}^{\alpha}(\mu, \cdot)$ is $\mathcal{S}^{\alpha}$-measurable. Arguing as in the proof of Theorem 5.7, we observe that condition (6) of Theorem 4.2 is satisfied, again using the notion of enlargement of (2.7). We can apply Theorem 4.2, that concludes the proof.

Remark 8. Let $\mathcal{F}_{b}^{\prime}$ be the family of balls introduced in Remark7, Then in the assumptions of either Theorem 5.7 or Theorem 5.8 we actually have $\left(\mathcal{F}_{b}\right)_{\mu, \zeta_{b, \alpha}}=\mathcal{F}_{b}^{\prime}$. As already pointed out in Remark 7, it holds $\mathcal{F}_{b}^{\prime}=\left(\mathcal{F}_{b}^{\prime}\right)_{\mu, \zeta_{b, \alpha}} \subset\left(\mathcal{F}_{b}\right)_{\mu, \zeta_{b, \alpha}}$. Conversely, any set of $\left(\mathcal{F}_{b}\right)_{\mu, \zeta_{b, \alpha}}$ must differ from a point, due to the absolute continuity of $\mu \mathrm{L} A$ with respect to $\mathcal{S}^{\alpha} L A$, hence the opposite inclusion holds. Thus, in both Theorems 5.7 and 5.8 the assumption (3) can be replaced by the condition that $\mathcal{F}_{b}^{\prime}$ covers $A$ finely.

\section{REFERENCES}

[1] Luigi Ambrosio and Bernd Kirchheim. Rectifiable sets in metric and Banach spaces. Math. Ann., 318(3):527-555, 2000.

[2] Herbert Federer. Geometric Measure Theory. Springer, 1969.

[3] Gerald B. Folland and Elias M. Stein. Hardy Spaces on Homogeneous groups. Princeton University Press, 1982.

[4] B. Franchi, R. P. Serapioni, and F. Serra Cassano. Area formula for centered Hausdorff measures in metric spaces. Nonlinear Anal., 126:218-233, 2015. 
[5] Mikhael Gromov. Carnot-Carathéodory spaces seen from within. In Sub-Riemannian geometry, volume 144 of Progr. Math., pages 79-323. Birkhäuser, Basel, 1996.

[6] Antoine Julia, Sebastiano Nicolussi Golo, and Davide Vittone. Area of intrinsic graphs and coarea formula in carnot groups. arXiv:2004.02520, April 6, 2020.

[7] Bernd Kirchheim. Rectifiable metric spaces: local structure and regularity of the Hausdorff measure. Proc. Amer. Math. Soc., 121(1):113-123, 1994.

[8] Valentino Magnani. On a measure theoretic area formula. Proc. Roy. Soc. Edinburgh Sect. A, 145:885-891, 2015.

[9] Valentino Magnani. A new differentiation, shape of the unit ball, and perimeter measure. Indiana Univ. Math. J., 66(1):183-204, 2017.

[10] Valentino Magnani. Some remarks on densities in the Heisenberg group. Ann. Acad. Sci. Fenn. Math., 42(1):357-365, 2017.

[11] Valentino Magnani. Towards a theory of area in homogeneous groups. Calc. Var. Partial Differential Equations, 58(3):58:91, 2019.

[12] Valentino Magnani, Eugene Stepanov, and Dario Trevisan. A rough calculus approach to level sets in the Heisenberg group. J. Lond. Math. Soc. (2), 97(3):495-522, 2018.

[13] D. Preiss. Geometry of measures in $\mathbf{R}^{n}$ : distribution, rectifiability, and densities. Ann. of Math. (2), 125(3):537-643, 1987.

[14] D. Preiss and J. Tišer. On Besicovitch's $\frac{1}{2}$-problem. J. London Math. Soc. (2), 45(2):279-287, 1992.

Giacomo Maria Leccese, SiSSA, Via Bonomea 265, 34136 Trieste, Italy

Email address: giacomomaria.leccese@sissa.it

Valentino Magnani, Dip.to di Matematica, Università di Pisa, Largo Bruno Pontecorvo 5, I-56127, Pisa

Email address: valentino.magnani@unipi.it 